\title{
ENERGY APPROACH FOR MULTIPLE CRACKING IN BEAMS MADE OF QUASI- BRITTLE MATERIALS UNDER PURE BENDING
}

\author{
TIANSHENG SHI ${ }^{*} \&$ CHRISTOPHER K.Y. LEUNG ${ }^{\dagger}$ \\ Hong Kong University of Science and Technology \\ Hong Kong, China \\ e-mail: tshiaa@ust.hk \\ ${ }^{\dagger}$ Hong Kong University of Science and Technology \\ Hong Kong, China \\ e-mail: ckleung@ust.hk
}

Key words: Energy Minimization, Multiple Cracking, Crack Pattern, Cohesive fracture

\begin{abstract}
For beams made of quasi-brittle materials, the moment can increase after a crack starts to propagate so the formation of multiple cracks is possible. Here, an energy minimization based method is proposed to determine the pattern of multiple cracking in such beam under pure bending. The fracture behavior of quasi-brittle material is described through cohesive zone model (CZM) with a linear softening law. Assuming the presence of a periodic and parallel crack array in the beam, the effects of crack spacing and beam curvature on the total energy per unit length of the member is obtained through finite element (FE) analysis. In the FE model, only a representative segment of the beam with a single crack needs to be considered, so the modeling is simple and computationally efficient. After performing a series of analyses on a bending beam, the crack spacing corresponding to the lowest energy is found at different curvatures and the relation between this crack spacing and the curvature is used to describe the crack pattern evolution of the member. Normalization is conducted and the effect of various parameters (tension softening law, elastic modulus of material and height of beam) on the cracking process is also studied. The results indicate that multiple cracking behavior in beams made of a linear softening material is determined by only one dimensionless parameter-brittleness number. As the crack pattern derived from the proposed energy approach has a sound physical basis, it can potentially be used as a reference for checking if the multiple cracking pattern obtained by other computational methods is proper or not.
\end{abstract}

\section{INTRODUCTION}

Quasi-brittle materials such as concrete and fiber reinforced concrete are widely used in civil engineering. For such material of low tensile strength, cracking is an important issue because cracks affect both the mechanical behavior of the structure and its serviceability. Large crack opening may also lead to durability problem. Methods to predict the crack process and to control the crack are hence very important.
In some cases, multiple cracks occur very close to each other. With the interaction among neighboring cracks, the crack propagation process usually involves stability problem. Many works have been done to predict the complex crack pattern in such a situation. Nemat-nasser et al. (1978) and Bazant et al. (1979) used bifurcation analysis to study selective propagation of multiple crack in rock under cooling. Bazant and Wahab (1980) study the pattern of bending 
cracks in concrete reinforced by bars with a similar approach. Jenkins (2005) proposed an energy minimization method to predict both the spacing and penetration of crack in brittle slab under shrinkage. In these studies, analysis is performed according to linear fracture mechanics which is not appropriate for quasibrittle material, for which nonlinear fracture models, e.g. smeared crack model by Bazant and Oh (1983) or fictitious crack model (discrete crack model) by Hillerborg et al. (1976) should be used.

Horii and Inoue (1997) studied the pattern evolution of multiple cracks in concrete beam using smeared crack model. They judged the instability by the sign of Hessian matrix and showed that consideration of instability is important for capturing the ultimate strength and ductility of the member. Shi et al. (2001) analyzed multiple cracking problem of concrete beam adopting discrete crack model. They proposed a scheme that only one crack is active in one calculation and selected the case corresponding to the lowest load. Amarasiri and Kodikara (2014) studied multiple cracking under shrinkage (cooling) using discrete crack model, without any explicit consideration of bifurcation. These works are preliminary, and particularly the force criterion adopted by Shi et al. (2001) is doubtful, so further work should be done to study the multiple cracking problem of quasi-brittle material.

Here, an energy minimization based method is presented to study the multiple cracking problem of bending beam made of quasi-brittle material. In the following sections a simple FE model with proper boundary conditions is first created and implemented into the FE software ABAQUS. After detailed discussions of the results of the case with linear tensile softening law, a parametric study is performed to see if it is possible to identify a single dimensionless parameter governing the multiple cracking process.

\section{METHODOLOGY AND FE MODEL}

\subsection{Methodology}

The minimum energy principle is widely used in physics, chemistry, biology and engineering to determine the stability of a body or structure. Here for the cracked beam, the total energy considered includes the elastic potential energy of uncracked elastic part as well as energy associated with the crack.

The expression for the total energy of the cracked beam is:

$$
\begin{gathered}
U=\int_{\Omega} U_{e}(\boldsymbol{\varepsilon}) d \Omega+\int_{\Gamma} \Phi(\delta) d \Gamma \\
U_{e}(\boldsymbol{\varepsilon})=\int \boldsymbol{\sigma}_{e}{ }^{T} d \boldsymbol{\varepsilon}=\int \boldsymbol{\varepsilon}^{T} \boldsymbol{C} d \boldsymbol{\varepsilon}=\frac{1}{2} \boldsymbol{\varepsilon}^{T} \boldsymbol{C} \boldsymbol{\varepsilon} \\
\Phi(\delta)=\int \sigma(\delta) d \delta
\end{gathered}
$$

In the above, $U$ is the total energy of the cracked beam; $U_{e}$ is the strain energy density of the elastic part; $\Phi(\delta)$ is the energy associated with the crack including both the reversible energy and the irreversible part. $\delta$ is the width of crack opening; $\sigma(\delta)$ is the crack bridging law, $\Omega$ is the volume of elastic part; $\Gamma$ is the area of crack surface; $\boldsymbol{C}$ is the stiffness matrix of material; $\boldsymbol{\varepsilon}$ is the engineering strain vector; $\sigma_{\boldsymbol{e}}$ is the stress vector.

Following previous works of Nemat-nasser et al. (1978), Bažant et al. (1979) and Jenkins (2005), a periodic array of parallel cracks is assumed to form in the beam. Then the total energy $U$, which is a unique function (except in the snap back stage) of the crack spacing $(S p)$ and curvature $(\kappa)$, can be expressed as:

$$
U=U(S p, \kappa)
$$

At each deformation level (or curvature), the optimal crack spacing $\left(S p_{\text {opt }}\right)$ can be obtained from the minimum point of the computed energy landscape $U(S p, \kappa)$. This can be expressed as:

$$
S p_{\text {opt }}(\kappa)=\operatorname{argmin}_{S p}\{U(S p, \kappa)\}
$$

The energy $U$ is evaluated by FE method. To assess the total energy of the assumed periodically cracked system, the problem is reformulated as follows: one representative segment with a cracked section in its middle is selected from a beam with periodic crack pattern of certain crack spacing. Bending moment is then applied to this segment. To calculate the energy of the segment under 
bending moment, a beam consisting of the chosen segment in the middle and two rigid parts on each side is built. The beam is simply supported and loaded in four-point bending, with two loading points equidistant from the cracked section and positioned on the rigid part. Loading is applied through displacement control. Due to symmetry, only half of the beam is analyzed. This simplification process is schematically illustrated in Figure 1. By specifying different crack spacing, i.e. changing the width of elastic segment in $\mathrm{FE}$ model, a set of FE analyses are performed. From these FE results, the total energy of each case (corresponding to different crack spacing) can be determined for different deformation levels.

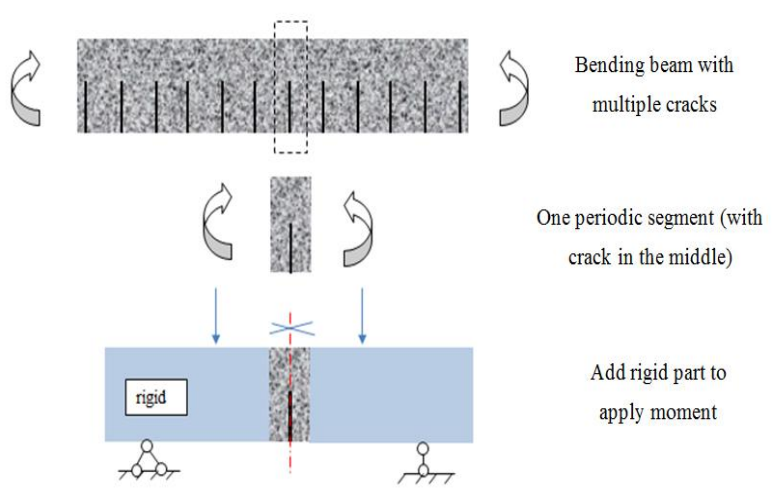

Figure 1: Model simplification

For better comparison among cases with different crack spacing, the total energy and deformation are normalized by crack spacing, thus the relationship of energy per unit length of beam and averaged curvature is obtained as follows:

$$
\begin{aligned}
\underline{U} & =\frac{U}{S p} \\
\kappa & =\frac{\Delta \phi}{S p}
\end{aligned}
$$

$U$ is total energy of the bending segment; $\underline{U}$ is the energy per unit length of beam; $\Delta \phi$ is relative rotation of the two sides of the elastic segment that is under bending. Because the difference between energy per unit length of different cases is relatively small compared to the value of the total energy per unit length, it is not very obvious when plotted in a figure.
To clearly present the results, the relative energy per unit length is obtained by subtracting that of a benchmark case from all other cases.

\subsection{FE model}

The above method is implemented into the ABAQUS FE package, and the FE model, consists of three parts: the elastic part, the rigid part and the crack. The beam height is $500 \mathrm{~mm}$ and the span is $2940 \mathrm{~mm}$. The chosen height is sufficient for observing the detailed development of the complete multiple cracking stages.

In the proposed FE model, both the elastic part and the rigid part are modeled with fournode bilinear plane stress element. Full Gauss integration (4 integration points) is employed for the elements. The mesh size is $10 \mathrm{~mm}$ by $10 \mathrm{~mm}$, which is sufficient according to preliminary mesh convergence study. To properly model the cracking behavior of quasibrittle material, the cohesive zone model (Barenblatt, 1959; Dugdale, 1960) is used. In this work, four-nodes two-dimension cohesive element is chosen, and the size is $10 \mathrm{~mm}$ by $1 \mathrm{~mm}$ (thickness). For such element, two Gauss points are used for integration.

\subsection{Material models}

For the elastic and rigid parts, the elastic moduli are $20 \mathrm{GPa}$ and $200,000 \mathrm{GPa}$, while the Poisson's ratio are 0.2 and 0.3 , respectively. Note that the Poisson's ratio of the rigid part does not affect the result because it is almost un-deformed during loading.

In this study, the linear softening law is adopted for the crack. The behavior of the crack is defined through the relation of stress vs. crack opening displacement as:

$$
\sigma(\delta)=\left\{\begin{aligned}
\frac{\delta_{u}-\delta}{\delta_{u}} f_{t} ; & 0 \leq \delta<\delta_{u} \\
0 ; & \delta \geq \delta_{u}
\end{aligned}\right.
$$

where $\sigma$ is the traction stress between the crack surface; $\delta$ is the traction displacement; $f_{t}$ is the tensile strength of quasi-brittle material.

The material parameters of the crack are selected as follows: $f_{t}=3 \mathrm{MPa}$; The other 
important parameter of the constitutive law, $\delta_{u}$, is changed from case to case, with details to be discussed later.

In our FE analysis, the constitutive response of the crack is defined using a tractionseparation description built in the cohesive element of ABAQUS. Compared with the constitutive law of crack, an elastic stage with a high elastic modulus is added to CZM. The initial elastic modulus is set to be $200 \mathrm{GPa}$, which is sufficiently large (10 times) compared with that of elastic part $(20 \mathrm{GPa})$.

The mode I and mode II fracture are uncoupled in the chosen cohesive element model. Because only mode I type of crack is studied here, the mode II deformation of crack is properly constrained to avoid unexpected deformation modes of the cohesive element.

For fracture problem, snap back instability (Crisfield, 1986) can occur in some cases, which prohibits further analysis. To improve the convergence of the FE model, a proper but small regulation viscosity is added to the constitutive law of CZM. In our study, the value of viscosity is chosen to be $1 \mathrm{e}-5$. From earlier analyses, this value can ensure convergence but it is sufficiently small to have little effect on the overall result.

\subsection{Normalization}

As only one set of member dimensions is employed in the analysis, it is desirable to normalize the results so they can apply to other cases. To do normalization, the geometry (height of beam) and material parameters (elastic modulus, strength and softening law) are also taken as variables, in addition to $S p$ and $\kappa$ mentioned above. Thus the total energy is dependent on these parameters: $E, h, f_{t}, \delta_{u}$, $S p$ and $\kappa$, and the function of total energy per unit length of beam can be fully expressed as:

$$
\underline{U}=\Psi\left(E, h, f_{t}, \delta_{u}, S p, \kappa\right) / S p
$$

From the Buckingham $\pi$ theorem (Buckingham, 1914), one way of normalization is:

$$
U^{*}=\underline{U} / \underline{U}_{0} ; \underline{U}_{0}=f_{t}^{2} b h /(6 E)
$$

$$
\begin{gathered}
\kappa^{*}=\kappa / \kappa_{0} ; \kappa_{0}=2 f_{\imath} /(\mathrm{E} h) \\
\delta_{u}{ }^{*}=\delta_{u} / h \\
S p^{*}=S p / h
\end{gathered}
$$

where $h$ is the height of the beam; $E$ is the elastic modulus; $\kappa_{0}, \underline{U}_{0}$ are the curvature and energy per length of beam when the tensile strength $\left(f_{t}\right)$ of material is reached. In addition, $E$ can be normalized by $f_{t}$ as: $E / f_{t}$

The function of total energy after normalization is:

$$
U^{*}=\Phi\left(E / f_{t}, \delta_{u}{ }^{*}, S p^{*}, \kappa^{*}\right)
$$

Then the normalized optimal crack spacing is:

$$
S p^{*}=\operatorname{argmin}\left\{\Phi\left(E / f_{t}, \delta_{u}^{*}, S p^{*}, \kappa^{*}\right)\right\}
$$

Other physical quantities are also normalized as follows:

$$
\begin{gathered}
M^{*}=M / M_{0} ; M_{0}=f_{t} b h^{2} / 6 \\
U_{\text {visco }}^{*}=\underline{U}_{\text {visco }} \underline{U}_{0}
\end{gathered}
$$

$\underline{U}_{\text {visco }}$ is the energy per unit length of beam dissipated through viscosity; $M$ is the bending moment applied.

\section{RESULT AND DISCUSSION}

A case with parameter $\delta_{u}{ }^{*}=6 \mathrm{e}-3$ is analyzed here and results are discussed in details. For this set of crack parameters, 23 cases with different spacing $\left(S p{ }^{*}=0.04,0.08\right.$, $0.12,0.16,0.2,0.24,0.28,0.32,0.36,0.4$, $0.44,0.48,0.6,0.72,0.8,0.88,11.12,1.2,1.4$, $1.6,1.8,2)$ are analyzed.

\subsection{Individual crack initiation and propagation}

The load-displacement curve as well as crack propagation process of a typical case $\left(S p^{*}=0.4\right)$ are shown in Figure 2. 


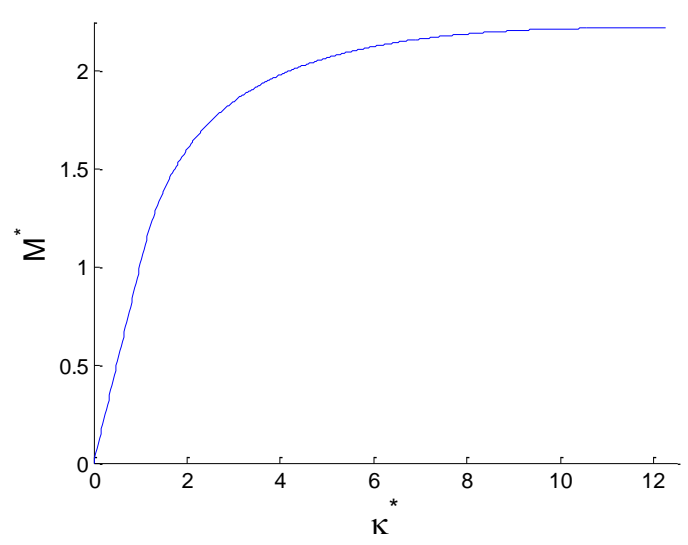

(a) Moment vs. curvature

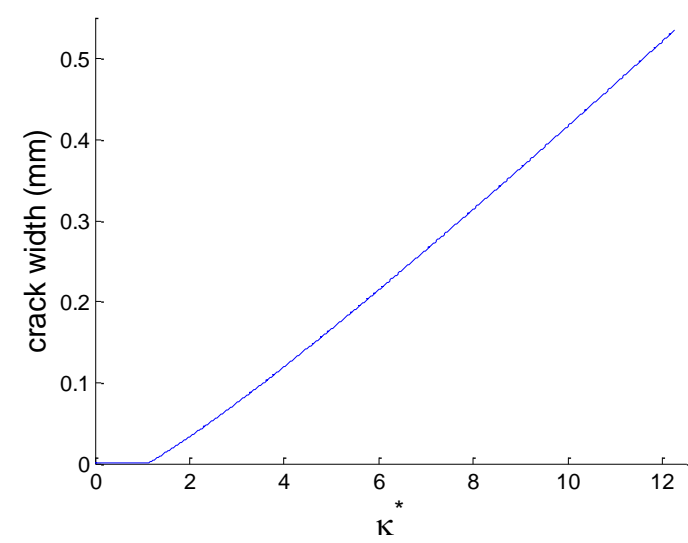

(b) Crack width vs. curvature

Figure 2: Load-displacement curve and crack propagation process

From the load-curvature curve in Figure 2 (a), a sudden change of slope is found when the normalized curvature $\left(\kappa^{*}\right)$ reaches a value of 1.1. This point corresponds to a rapid increase in crack width at the bottom of the member with the curvature, as shown in Figure 2 (b). As $\kappa^{*}>1.1$, the crack width develops almost linearly with the beam curvature. It is interesting to note that the crack only opens very slightly at $\kappa^{*}=1$ when the stress at the bottom reaches the value of $f_{t}$, the tensile strength of the material.

\subsection{Optimal crack spacing}

The relative energy vs. curvature curves for each case with different crack spacing are collected and compared as shown in Figure 3. The benchmark case chosen for subtraction is the case with normalized crack spacing of 0.4. The overall shape of these curves are complicated but can be viewed as generally a piecewise parabolic shape because energy is a parabolic function of curvature. At small curvatures $\left(\kappa^{*}\right.$ in the range of around 1.2 3), all curves concave downwards. At large curvatures $\left(\kappa^{*}\right.$ in the range of 4 to 8 ), the curves of larger crack spacing $\left(S p^{*}=1.6\right)$ concave downwards while those for small crack spacing concave upwards. The change in shape is because different fractions of elastic energy and fracture energy are present in the total energy for different crack spacing at different loading stages, as the crack widens and propagates.

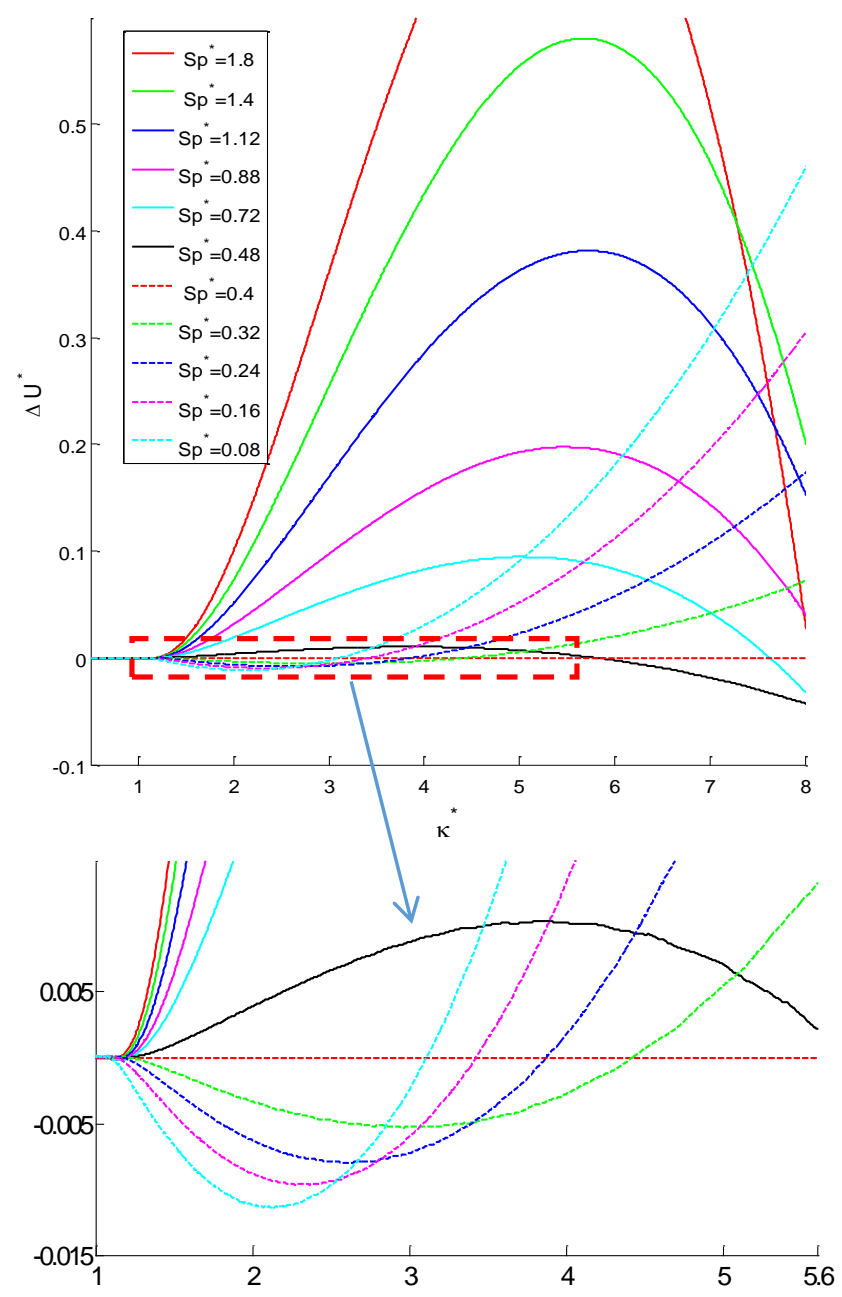

Figure 3: Relative energy-curvature curves

From Figure 3, the relative positions of these curves are found to change when the member goes through different stages, including crack initiation, crack propagation and final localization, with increasing curvature. When cracking initiates, the energy- 
curvature curves for various crack spacing start to deviate from each other. During the final localization stage, the energy corresponding to the largest crack spacing is lower than that of smaller spacing. (Note: a very large spacing which is many times the member dimension implies the propagation of a single crack in practice.) Between crack initiation stage and final localization stage is the multiple crack propagation stage. During this stage, the crack spacing with the lowest energy changes with increasing curvature, indicating that the multiple cracks interact with each other and the crack pattern tend to change in order to achieve the lowest energy state.

From these curves, the energy landscape for the bending problem of quasi-brittle material is constructed and plotted in Figure 4. Even without any smoothening manipulation in MATLAB (as the landscape is plotted with "surf" and "shading interp" commands only), the surface of energy landscape of this problem is rather smooth. It should also be noted that at each deformation level, there is only one local or global minimum, rather than several minimum values.

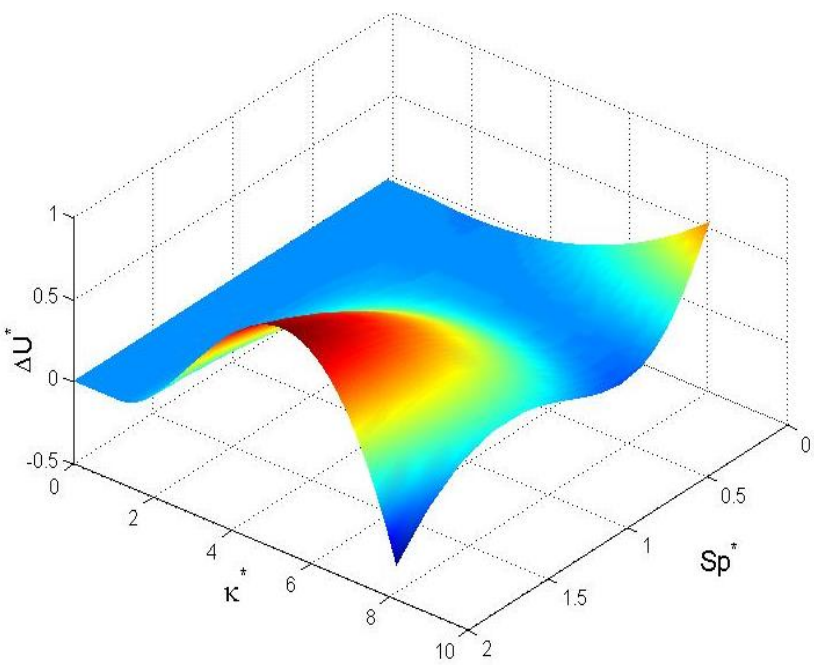

Figure 4: Landscape of energy

From the energy landscape, the relation of relative energy and crack spacing at different deformation levels (e.g., $\kappa^{*}=1,2,3,4,5,6,7$ and 8) can be found, as shown in Figure 5. The curves are rather smooth.

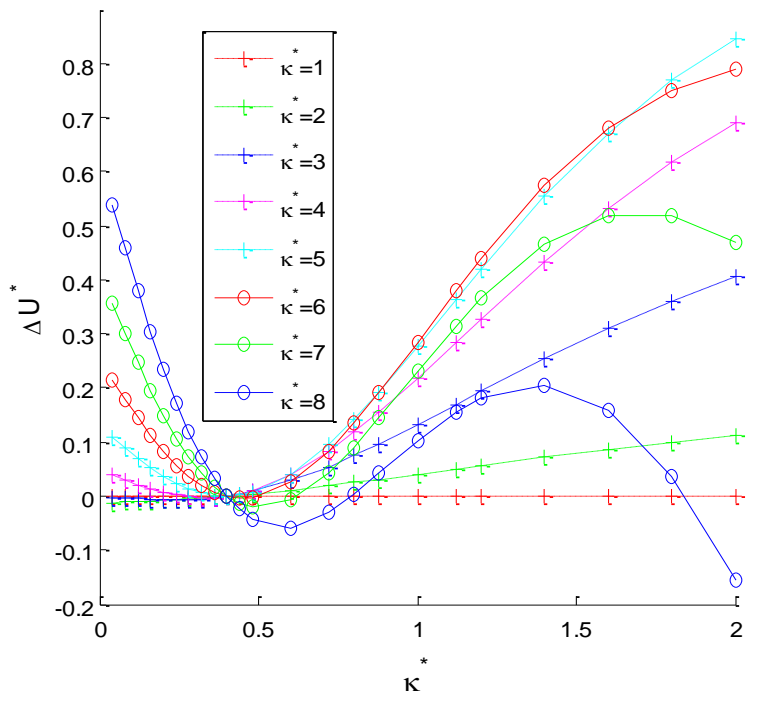

Figure 5: Comparison of relative energy at different crack spacing

To find the evolution of optimal crack spacing with curvature, the crack spacing corresponding to minimum energy at different curvatures is obtained and plotted in Figure 6. Before the crack opens significantly $\left(\kappa^{*}=1.1\right)$, there is theoretically no difference between the energies of different crack spacing but numerical error will lead to erratic variations in the $S p^{*}$ vs $\kappa$ curve. To avoid confusion, the early part of the curve is removed from the figure. Following the work of Jenkins (2005) which employs the optimal crack spacing to describe the multiple cracking process, Figure 6 can be interpreted in the following manner: After crack initiation, many cracks are formed in the specimen at very small crack spacing. The cracks are constrained to propagate at this spacing until $\kappa^{*}$ reaches a value of 2.66 , when the optimal crack spacing becomes larger which indicates selective propagation of the cracks. With increasing curvature, this process repeats with decreasing number of propagating cracks. At $\kappa^{*}=7.85$, the optimal normalized crack spacing jumps to 2 (the largest crack spacing studied, which is a very large value compared with the depth of beam). This indicates the localization of multiple cracks into a single crack. 


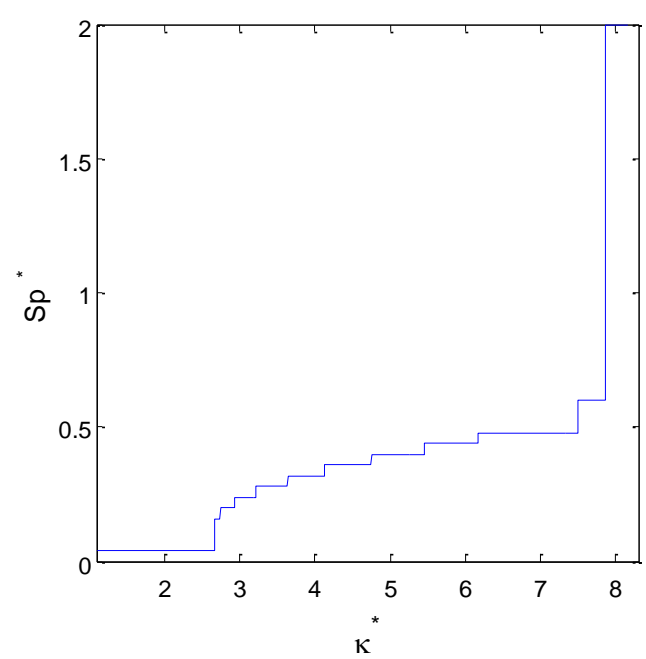

Figure 6: Optimal crack spacing at different deformation level

\section{PARAMETRIC STUDY}

As shown in eq. (9) of section 2.4, the crack pattern or optimal crack spacing is related to $E / f_{t}, \delta_{u}{ }^{*}\left(\right.$ or $\left.\delta_{u} / h\right)$ and $\kappa^{*} . E / f_{t}$ and $\delta_{u} / h$ reflect geometric and material information of the beam. Parametric study is conducted to find whether these two parameters can be combined into one parameter determining the behavior of cracking behavior.

\subsection{The chosen combinations of parameters}

The combinations are listed in Table 1 as:

Table 1: Different combinations of parameters for analyses

\begin{tabular}{ccccccc}
\hline NO. & $\begin{array}{c}E \\
(\mathrm{Gpa} \\
)\end{array}$ & $\begin{array}{c}f_{t} \\
(\mathrm{Mpa})\end{array}$ & $\begin{array}{c}\delta_{u} \\
(\mathrm{~mm})\end{array}$ & $\begin{array}{c}h \\
(\mathrm{~mm})\end{array}$ & $E / f_{t}$ & $\delta_{u} / h$ \\
\hline $\mathrm{C} 1$ & 20 & 3 & 3 & 500 & 6667 & 0.006 \\
\hline $\mathrm{C} 2$ & 20 & 3 & 6 & 500 & 6667 & 0.012 \\
\hline $\mathrm{C} 3$ & 20 & 3 & 12 & 500 & 6667 & 0.024 \\
\hline $\mathrm{C} 4$ & 20 & 3 & 24 & 500 & 6667 & 0.048 \\
\hline $\mathrm{C} 5$ & 10 & 3 & 12 & 500 & 3333 & 0.024 \\
\hline $\mathrm{C} 6$ & 5 & 3 & 12 & 500 & 1667 & 0.024 \\
\hline $\mathrm{C} 7$ & 20 & 3 & 12 & 250 & 6667 & 0.048 \\
\hline
\end{tabular}

\subsection{Result of parametric study}

Through analyses using energy approach proposed above, the result of most favorable crack spacing vs curvature (both normalized) is shown in Figure 7.

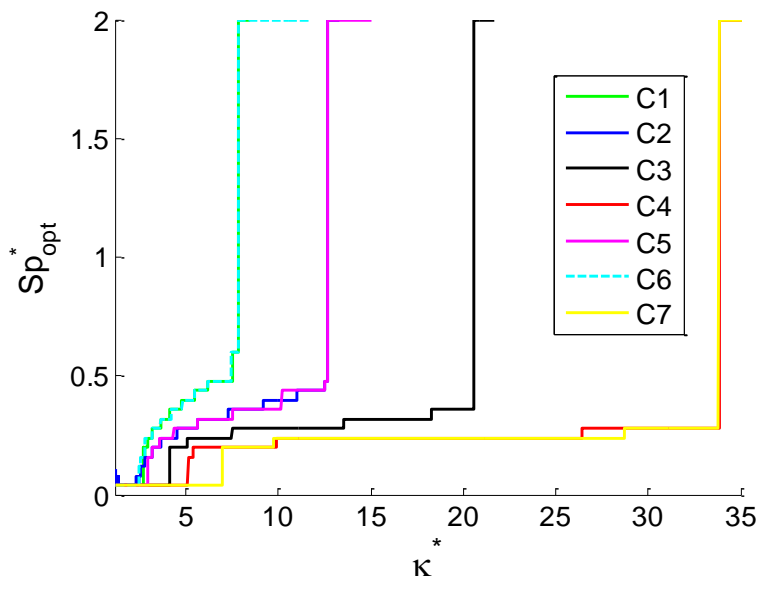

Figure 7: Crack spacing evolution for different parameter combinations

From Figure 7, it is found that the curves for combinations $\mathrm{C} 1, \mathrm{C} 2$ and $\mathrm{C} 4$ are very close to those for $\mathrm{C} 6, \mathrm{C} 5$ and $\mathrm{C} 7$ respectively. As shown in Table 2, for curves which are essentially overlapping, the values of $E \delta_{u} / f_{t} h$ are the same. $E \delta_{l} / f_{t} h$ can hence be considered as a parameter governing the multiple cracking process. This parameter is actually half of the reciprocal of the brittleness number proposed by Bazant and Planas (1997) for study of size effect in structural design. From Figure 7, we can see that a member with lower brittleness (higher $E \delta_{u} / f_{t} h$ ) shows crack localization at higher curvature, which is consistent with intuition.

Table 2: Combinations and new parameter

\begin{tabular}{cccc}
\hline$N O$. & $E / f_{t}$ & $\delta_{u} / h$ & $E \delta_{u} / f_{t} h$ \\
\hline $\mathrm{C} 1$ & 6666.7 & 0.006 & 40 \\
\hline $\mathrm{C} 2$ & 6666.7 & 0.012 & 80 \\
\hline $\mathrm{C} 3$ & 6666.7 & 0.024 & 160 \\
\hline $\mathrm{C} 4$ & 6666.7 & 0.048 & 320 \\
\hline $\mathrm{C} 5$ & 3333.3 & 0.024 & 80 \\
\hline $\mathrm{C} 6$ & 1666.7 & 0.024 & 40 \\
\hline $\mathrm{C} 7$ & 6666.7 & 0.048 & 320 \\
\hline
\end{tabular}

\section{CONCLUSIONS}

In this study, an energy minimization based method is first developed to predict the multiple cracking pattern in quasi-brittle beams through a series of analyses on simple FE models. The linear softening case is studied 
here and it is found that the crack spacing becomes larger and larger until failure occur with a localized crack. Through normalization and parametric study, one dimensionless parameter, which is of the same form as the brittleness number, is found to govern the behavior of multiple cracking process of quasibrittle beam.

Though only the bending beam is studied here, the same method can be used to study multiple cracking of quasi-brittle material due to shrinkage and cooling as well. With sound physical basis, the energy approach can also be potentially used as a reference to verify other numerical method.

\section{REFERENCES}

[1] Buckingham, E. (1914). On physically similar systems: illustrations of the use of dimensional equations. Physical Review, 4(4), 345-376.

[2] Barenblatt, G. (1959). The formation of equilibrium cracks during brittle fracture. General ideas and hypotheses. Axiallysymmetric cracks. Journal of Applied Mathematics and Mechanics, 23(3), 622636.

[3] Dugdale, D. S. (1960). Yielding of steel sheets containing slits. Journal of the Mechanics and Physics of Solids, 8(2), 100-104.

[4] Hillerborg, A., Modéer, M., \& Petersson, P. E. (1976). Analysis of crack formation and crack growth in concrete by means of fracture mechanics and finite elements. Cement and concrete research, 6(6), 773781.

[5] Nemat-Nasser, S., Keer, L. M., \& Parihar, K. S. (1978). Unstable growth of thermally induced interacting cracks in brittle solids. International Journal of Solids and Structures, 14(6), 409-430.

[6] Bažant, Z. P., Ohtsubo, H., \& Aoh, K. (1979). Stability and post-critical growth of a system of cooling or shrinkage cracks. International Journal of Fracture, 15(5), 443-456.
[7] Bažant, Z. P., \& Wahab, A. B. (1980). Stability of parallel cracks in solids reinforced by bars. International Journal of Solids and Structures, 16(2), 97-105.

[8] Bažant, Z. P., \& Oh, B. H. (1983). Crack band theory for fracture of concrete. Matériaux et construction, 16(3), 155177.

[9] Crisfield, M. A. (1986). Snap-through and snap-back response in concrete structures and the dangers of underintegration. International Journal for Numerical Methods in Engineering, 22(3), 751-767.

[10] Horii, H., \& Inoue, J. New directions: analysis of cracking localization and ultimate strength of structures in civil engineering. Advances in Fracture Research, Proc. of the Ninth Int. Conf. on Fracture, 1-5 April 1997, Sydney, Australia, Pergamon, Vol. 4 (1997) 18731884.

[11] Bazant, Z. P., \& Planas, J. (1997). Fracture and size effect in concrete and other quasibrittle materials (Vol. 16). CRC press. 319-322

[12] Shi, Z., Ohtsu, M., Suzuki, M., \& Hibino, Y. (2001). Numerical analysis of multiple cracks in concrete using the discrete approach. Journal of Structural Engineering, 127(9), 1085-1091.

[13] Jenkins, D. R. (2005). Optimal spacing and penetration of cracks in a shrinking slab. Physical Review E, 71(5), 056117.

[14] Amarasiri, A. L., \& Kodikara, J. K. (2014). Effect of Characteristic Lengths of Fracture on Thermal Crack Patterns. International Journal of Geomechanics, 04014071. 